\title{
Mask-aligner Talbot lithography using a 193nm CW light source
}

\section{Andreas Vetter, Raoul Kirner, Dmitrijs Opalevs, Matthias Scholz, Patrick Leisching, et al.}

Andreas Vetter, Raoul Kirner, Dmitrijs Opalevs, Matthias Scholz, Patrick Leisching, Toralf Scharf, Wilfried Noell, Carsten Rockstuhl, Reinhard Voelkel, "Mask-aligner Talbot lithography using a 193nm CW light source," Proc. SPIE 10587, Optical Microlithography XXXI, 105870W (20 March 2018); doi: $10.1117 / 12.2296503$

SPIE. Event: SPIE Advanced Lithography, 2018, San Jose, California, United States 


\title{
Mask-aligner Talbot lithography using a $193 \mathrm{~nm} \mathrm{CW}$ light source
}

\author{
Andreas Vetter ${ }^{*}, \mathrm{a}, \mathrm{b}$, Raoul Kirner ${ }^{\mathrm{a}}$, Dmitrijs Opalevs ${ }^{\mathrm{c}}$, Matthias Scholz ${ }^{\mathrm{c}}$, Patrick Leisching ${ }^{\mathrm{c}}$, \\ Toralf Scharf ${ }^{\mathrm{d}}$, Wilfried Noell ${ }^{\mathrm{a}}$, Carsten Rockstuhl ${ }^{\mathrm{b}, \mathrm{e}}$, and Reinhard Voelkel ${ }^{\mathrm{a}}$ \\ asUSS MicroOptics SA, Rouges-Terres 61, CH-2068 Hauterive, Switzerland \\ ${ }^{\mathrm{b}}$ Institute of Nanotechnology (INT), Karlsruhe Institute of Technology, \\ Hermann-von-Helmholtz-Platz 1, 76344 Eggenstein-Leopoldshafen, Germany \\ 'TOPTICA Photonics AG, Lochhamer Schlag 19, 82166 Gräfelfing, Germany \\ dNanophotonics and Metrology Laboratory, École Polytechnique Fédérale de Lausanne \\ (EPFL), CH-1015 Lausanne, Switzerland \\ e Institute of Theoretical Solid State Physics (TFP), Karlsruhe Institute of Technology, \\ Wolfgang-Gaede-Strasse 1, 76131 Karlsruhe, Germany
}

\begin{abstract}
We present and discuss Talbot mask-aligner lithography, relying on a continuous wave laser emitting at $193 \mathrm{~nm}$ for the illumination. In this source, a diode laser at $772 \mathrm{~nm}$ is amplified by a tapered amplifier in master-oscillator power-amplifier configuration and frequency-quadrupled in two subsequent enhancement cavities using lithium triborate and potassium fluoro-beryllo-borate nonlinear crystals to generate the emission at $193 \mathrm{~nm}$. The high coherence and brilliance of such an illumination source is predestined for plane wave mask-aligner illumination, crucial in particular for high-resolution lithographic techniques such as Talbot lithography and phase-shift masks. Talbot lithography takes advantage of the diffraction effect to image periodic mask features via self-replication in multiples of the Talbot distance behind the photomask when exposed by a plane wave. By placing a photoresistcoated wafer in one of the Talbot planes, the mask pattern is replicated in the resist. Periodic patterns with diverse shapes are required for wire grid polarizers, diffraction gratings, and hole arrays in photonic applications as well as for filters and membranes. Using an amplitude mask with periodic structures, we demonstrate here with such a technique sub-micron feature sizes for various designs at a proximity gap of $20 \mu \mathrm{m}$.
\end{abstract}

Keywords: Diode lasers, laser beam shaping, UV lasers, lithography, nanostructure fabrication, Talbot lithography

\section{INTRODUCTION}

Up to now, for more than 50 years mask-aligner lithography has been relying mostly on high-pressure mercury lamps as illumination source, since the implementation of Excimer lasers ( $\mathrm{KrF}$ at $248 \mathrm{~nm}$ or $\mathrm{ArF}$ at $193 \mathrm{~nm}$ ) is complex, bulky, expensive, and requires regular maintenance as well as the use of toxic gases. ${ }^{1}$ Of course, all these aspects are rather detrimental. In contrast, diode lasers are more and more considered as illumination source for mask-aligner lithography, as they have much better characteristics in terms of durability, efficiency, and ease of implementation. ${ }^{2}$ This trend emerges in combination with the search of novel illumination sources emitting at lower wavelengths to increase the resolution in proximity lithography. The resolution in mask-aligner lithography is directly related to the illumination wavelength $\lambda$ and can be estimated by the relation

$$
\text { Resolution } \sim \sqrt{\lambda \cdot g}
$$

with the proximity gap $g$ between photomask and photoresist-coated wafer. The best resolution is obtained in contact lithography, i.e., with a vanishing gap. However, in production environments, mask contamination

\footnotetext{
*Further author information:

E-mail: andreas.vetter@suss.com, Telephone: +41 325664461

E-mail: andreas.vetter@kit.edu, Telephone: +49 72160846949
}

Optical Microlithography XXXI, edited by Jongwook Kye, Proc. of SPIE Vol. 10587, $105870 \mathrm{~W} \cdot(\complement) 2018$ SPIE $\cdot$ CCC code: $0277-786$ X/18/\$18 - doi: 10.1117/12.2296503 
reduces the reproducibility and requires frequent mask cleaning, which is highly undesirable. Therefore, the gap distance should not surpass a lower bound. Improving resolution, therefore, prompts for light sources that emit at lower wavelengths.

With the advent of the nonlinear potassium fluoro-beryllo-borate (KBBF) crystal, diode light sources achieving wavelengths below $200 \mathrm{~nm}$ have been demonstrated, relying on second-harmonic generation (SHG). ${ }^{3,4}$ First experimental results for contact and proximity lithography using such an illumination source have recently been published. ${ }^{5}$ Here, we demonstrate the suitability of this mask-aligner setup for Talbot lithography. Sub-micron structures relying on an amplitude mask and a proximity gap of $20 \mu \mathrm{m}$ are reported. In Talbot lithography, the diffraction of periodic mask features under plane wave illumination in the Fresnel regime is used to obtain a perfect self-image of the mask at certain distances. ${ }^{6,7}$ This Talbot distance $z_{\mathrm{T}}$ is a function of the illumination wavelength and the period of the mask pattern. With a photoresist-coated substrate located in such a Talbot plane, the image of the mask is replicated. However, highly collimated light is required to avoid lateral blurring of the aerial image. ${ }^{8}$ This is a complex task when using Excimer lasers as illumination source, as they suffer from being composed of many modes. ${ }^{9}$

Various aspects of Talbot lithography have been addressed in the past, ${ }^{10,11,12,13,14}$ including displacement Talbot lithography, ${ }^{15}$ self-assembled nanosphere masks, ${ }^{16}$ extreme ultraviolet Talbot lithography, ${ }^{17}$ and fractal and fractional ${ }^{18}$ as well as quantum Talbot effects. ${ }^{19,20}$ Most of the experimental examples rely on i-line maskaligner illumination at a wavelength of $365 \mathrm{~nm}$. However, a reduction in wavelength $\lambda$ is highly desired: Compared to traditional high-pressure mercury lamp illumination, lower wavelengths result in improved resolution $\Delta x$, increased Talbot distance ${ }^{7} z_{\mathrm{T}}=2 \Lambda^{2} / \lambda$, and enlarged depth of focus ${ }^{21} \mathrm{DOF} \sim 4 \Delta x^{2} / \lambda$ for a given mask structure period $\Lambda$.

The key to success for diode laser mask-aligner lithography is beam shaping, especially since plane wave illumination is required for Talbot lithography. In addition, emerging speckle patterns which arise from the high coherence of the source have to be effectively mitigated by introducing rotating shaped random diffusers in the beam path. ${ }^{5,22}$ Interference patterns emerging in the wafer plane are averaged over different speckle patterns over time. To emphasize these most crucial points, our approach to laser beam shaping in the deep ultraviolet (DUV) is discussed in the following.

In this manuscript, we first introduce our continuous wave $(\mathrm{CW})$ illumination source. Our beam shaping concept is discussed, relying on refractive microlens arrays, and plane wave illumination with uniform light distribution over the complete print field is demonstrated. Subsequently, we show simulations of the self-replication behind amplitude structures and print results. Finally, we draw a comparison to Talbot lithography relying on mercury lamp i-line illumination for the same amplitude mask structures.

\section{CW LIGHT SOURCE AT 193 NM FOR MASK-ALIGNER LITHOGRAPHY}

The setup of our CW diode laser source follows the master-oscillator power-amplifier (MOPA) scheme. A seed diode laser emits light at a wavelength of $772 \mathrm{~nm}$, which is magnified in a tapered amplifier (TA) to an optical power of $3 \mathrm{~W}$. Faraday isolators (FIs) are introduced to avoid parasitic back reflections. A first SHG stage contains a lithium triborate (LBO) nonlinear crystal inside a bow-tie cavity, stabilized using the Pound-Drever$\mathrm{Hall}^{23}$ scheme by piezoelectric elements.

The output at a wavelength of $386 \mathrm{~nm}$ is further upconverted in a second SHG stage to the target of $193 \mathrm{~nm}$ in the DUV, using a potassium fluoro-beryllo-borate (KBBF) crystal. To attain the necessary crystal axis for phase matching inside the crystal, $\mathrm{CaF}_{2}$ prisms are optically bonded on both sides. In this configuration, the spectral linewidth of the output is around $100 \mathrm{kHz},{ }^{4}$ orders of magnitude lower than for i-line illumination of standard high-pressure mercury arc lamps. The industrial grade laser source uses a crystal shifter for the KBBF and motorized mirrors to reach lifetimes up to $10000 \mathrm{~h}$.

In the described configuration, a maximum output power of up to $20 \mathrm{~mW}$ at $193 \mathrm{~nm}$ is possible, depending on the spot position on the KBBF crystal. For the exposures presented here, the laser output is stabilized at a level of $5 \mathrm{~mW}$. 


\section{BEAM SHAPING AND ILLUMINATION CONTROL}

For a uniform mask illumination, the elliptic beam profile of the laser has to be transformed to a flat-top profile. In addition, only small illumination angles can be used for Talbot lithography in order to obtain approximately plane wave illumination. Otherwise the image is blurred. ${ }^{8}$ Furthermore, the coherent nature of the light source has to be addressed, in order to prevent the formation of interference effects in the mask plane. Simultaneously, the power used for lithography has to be maximized.

We solve the task of laser beam shaping for Talbot lithography in an integral way, combining a rotating shaped random diffuser with an imaging microlens array (MLA) homogenizer, as depicted in Fig. 1. The high spatial and temporal coherence of the illumination source leads to speckle formation, which is highly undesirable. The rotating diffuser causes many statistically independent speckle patterns that are superimposed in the resist, effectively integrating over many inhomogeneous fields. This averaging smooths the illumination, effectively omitting the visibility of adverse coherence effects. ${ }^{5,22,24}$ For further discussion, please refer to Refs. 5 and 25.

The diverging beam behind the rotating diffuser is collimated on the subsequent Köhler integrator using a collector lens. The integrator consists of MLAs and a Fourier lens. Each MLA consists of cylindrical microlenses on both sides of a fused silica substrate, separated by one focal length. Two uncoated MLAs (SUSS MicroOptics) are used in crossed assembly to obtain a square flat-top in the mask plane. The Fourier lens behind the MLA leads to a superposition of all illuminated microlens channels in the mask plane. A front lens ensures telecentric illumination.

An iris aperture enables control over the illuminated area on the MLA and hence to choose the number microlenses contributing to the field distribution. Since the Fourier lens performs a Fourier transform in the mask plane, the illuminated area on the MLA corresponds to the illumination angles in the print field. By selectively blocking light, the angular spectrum can be shaped at will. This is often referred to as an illumination filter plate (IFP), and typically an integral part of tandem Köhler integrators (MO Exposure Optics ${ }^{\circledR}$ ). ${ }^{26,27}$ In this configuration, the first integrator ensures a uniform illumination of the second integrator, resulting in a uniform angular spectrum. Since we are interested in small illumination angles, we use only a single integrator. This reduces optical losses and increases the overall light efficiency of the system. By closing the iris aperture, a reduced number of channels is selected, corresponding to small illumination angles.

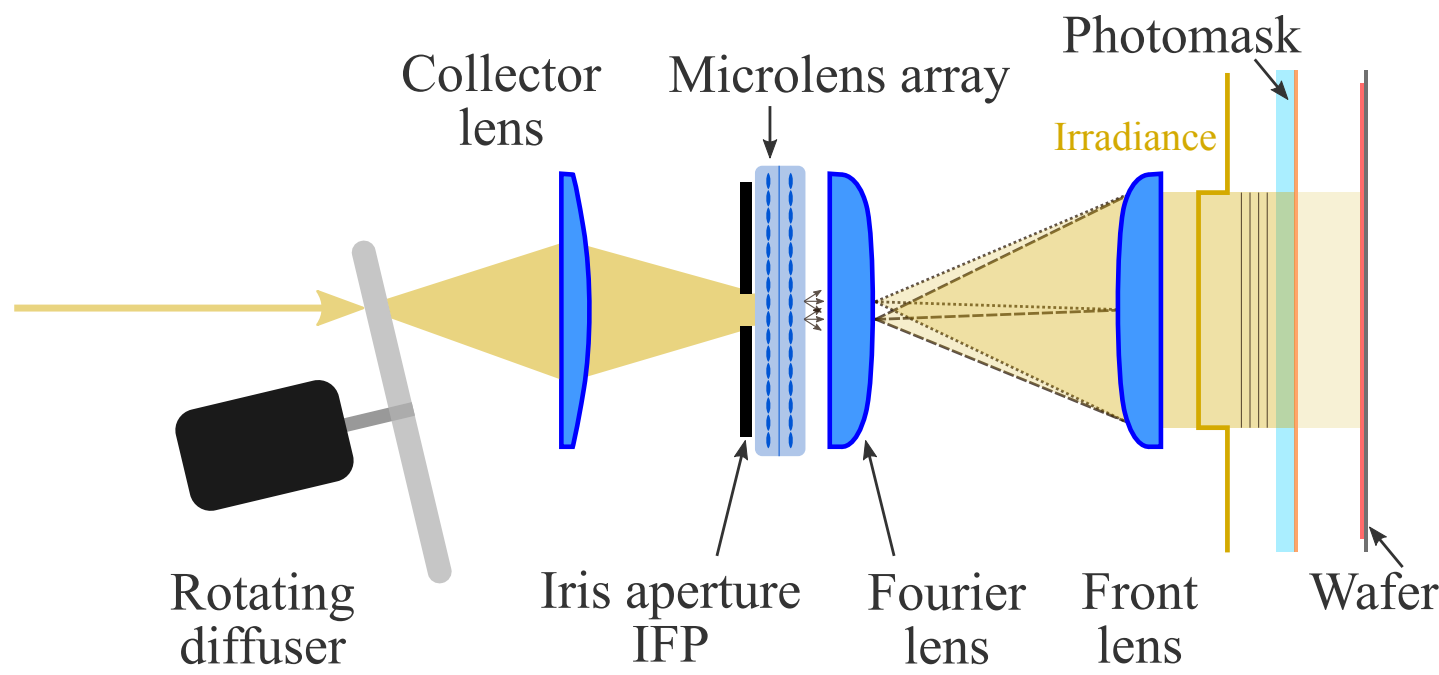

Figure 1: Optical beam shaping setup. The laser output passes a rotating shaped random diffuser, is collected in a collector lens, and homogenized via a Köhler integrator formed by two microlens arrays (MLAs). The maximum illumination angle is set by an iris aperture working as an illumination filter plate (IFP). Using this optical setup, a flat-top irradiance distribution is obtained in the mask plane. 

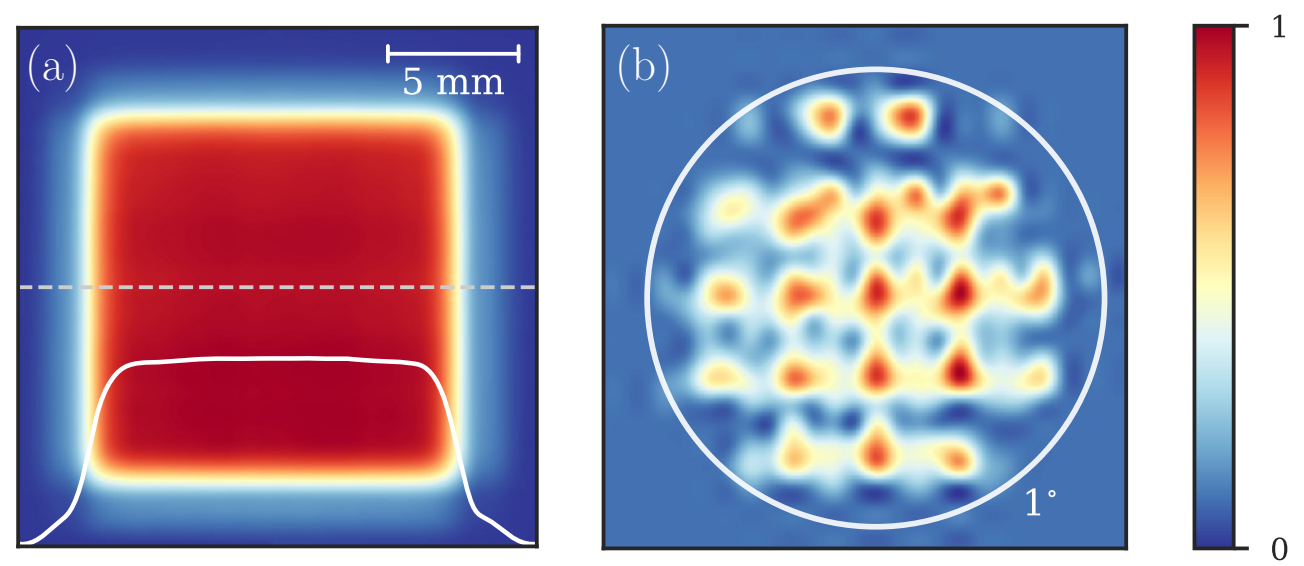

Figure 2: (a) Irradiance uniformity in the mask plane. The irradiance profile along the dashed line is depicted in white. A uniform irradiance uniformity is obtained on a square area. (b) Angular distribution of illumination angles in the mask plane. The white circle depicts an illumination angle of $1^{\circ}$. Individual microlens channels are visible.

We measure the uniformity of power in the field and in the angular spectrum using a customized beam profiler. It consists of a UV-sensitive photodiode (S1226-18BQ Si photodiode, Hamamatsu; photosensitivity 0.13 $\mathrm{A} \mathrm{W}^{-1}$ at $193 \mathrm{~nm})$ mounted on a xy precision stage. A laser-cut opening $(300 \mu \mathrm{m} \times 300 \mu \mathrm{m})$ is used as an aperture to select the area and to control the power incident on the photodiode. By scanning the mask plane, the irradiance is measured, as depicted in Fig. 2a. A highly uniform irradiance distribution is obtained, with a field size of approximately $15 \mathrm{~mm} \times 15 \mathrm{~mm}$.

The angular spectrum is obtained by inserting a plano-convex lens $(f=140 \mathrm{~mm})$ into the mask plane and measuring the irradiance at one focal distance, as depicted in Fig. 2b. The individual channels of the MLA are clearly visible. All channels used for the prints correspond to illumination under angles of less than $1^{\circ}$, as indicated by the white circle.

\section{SIMULATION OF TALBOT LITHOGRAPHY AT 193 NM}

For experimental prints, a chromium amplitude mask has been designed and fabricated. It includes several periodic structures, mainly of quadratic and circular shape. The Talbot planes behind the mask are located at integer multiples $n$ of the Talbot distance $z_{\mathrm{T}}$, given by ${ }^{7,13}$

$$
z_{\mathrm{T}}=n \cdot \frac{\lambda}{1-\sqrt{1-\frac{\lambda^{2}}{\Lambda^{2}}}}
$$

with the period $\Lambda$ of the mask features, the illumination wavelength $\lambda$, and an integer multiple $n$. In mask-aligner lithography, the period is typically larger than the wavelength, and Eq. 2 can be simplified to

$$
z_{\mathrm{T}} \simeq n \cdot \frac{2 \Lambda^{2}}{\lambda}
$$

in paraxial approximation. To maintain a high degree of accuracy, we use Eq. 2 for all calculations. By inverting Eq. 2, we obtain $\Lambda=1.39 \mu \mathrm{m}$ for a Talbot distance of $z_{\mathrm{T}}=20 \mu \mathrm{m}$ at $\lambda=193 \mathrm{~nm}$. This value is chosen since it allows sub-micron feature sizes, while the proximity gap between mask and wafer can still be set with ease. Small surface irregularities on the wafer do not result in contact between wafer and mask. Choosing different periods is possible by either changing the proximity gap or using higher Talbot planes. 
We simulate arrays of circles and squares using GenISys LAB, which solves the Rayleigh-Sommerfeld diffraction integral. The results are depicted in Fig. 3. The irradiance distribution for an array of circles with a period of $\Lambda=1.39 \mu \mathrm{m}$ is shown in Fig. 3a, together with the irradiance profile at a propagation distance of $20 \mu \mathrm{m}$. Figure $3 \mathrm{~b}$ shows the field distribution for an array of squares, here with a period of $\Lambda=0.99 \mu \mathrm{m}$, corresponding to a Talbot distance of $z_{\mathrm{T}}=10 \mu \mathrm{m}$. Accordingly, the second Talbot plane $n=2$ at $20 \mu \mathrm{m}$ can be used for printing. The irradiance distributions in the first and second Talbot plane for the square mask features are depicted in Figs. 3c and 3d.
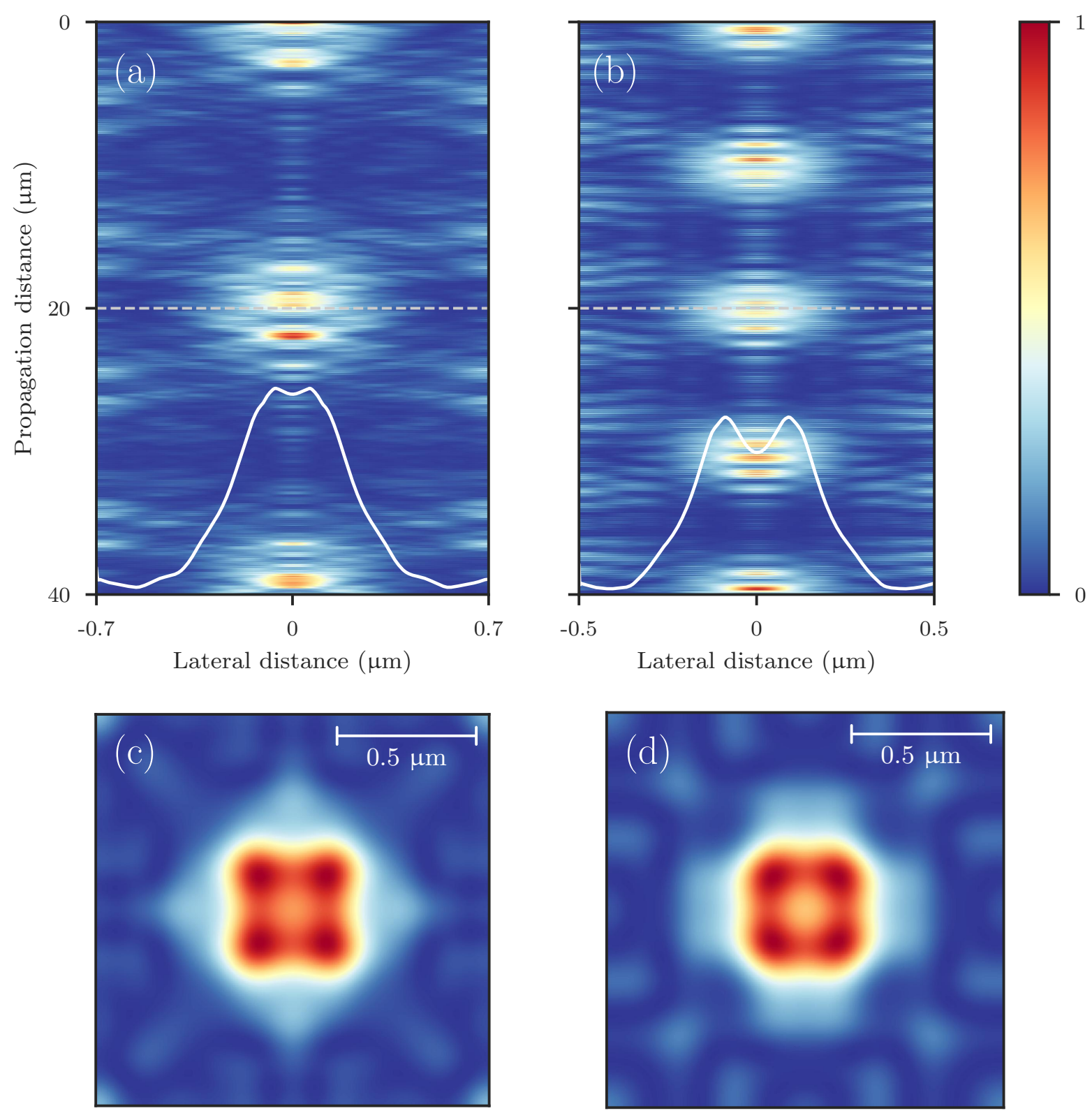

Figure 3: Simulated aerial image of periodic Talbot structures under plane wave illumination. (a) Array of circles with a period of $\Lambda=1.39 \mu \mathrm{m}$. (b) Array of squares with a period of $\Lambda=0.99 \mu \mathrm{m}$. (c) Irradiance distribution of the square array in the first Talbot plane. (d) Irradiance distribution of the square array in the second Talbot plane. The square irradiance profile is replicated in the Talbot planes with high contrast. 
As is clearly visible from the simulation, the irradiance distribution offers a high contrast, also for the second Talbot plane. This contrast decreases if more channels contribute to the illumination, i.e. additional plane waves under non-normal incidence contribute to the aerial irradiance formation. Consequently, for non-collimated illumination, there is a fundamental trade-off between light power in the mask plane and the maximum angle in the spectrum of plane waves, which defines the amount of blur in the Talbot planes.

\section{EXPERIMENTAL RESULTS}

With the optical setup implemented as described above, we perform lithographic prints for a field size of $15 \mathrm{~mm} \times 15 \mathrm{~mm}$ on Silicon substrates. We rely on the chemically amplified photoresist TOK TARF-P6239 ME, with the resist chemistry adapted from ArF Excimer lithographic systems. A prebake $\left(110^{\circ} \mathrm{C}, 1 \mathrm{~min}\right)$ is performed on a hotplate prior to the exposure, leading to a resist thickness of $\sim 120 \mathrm{~nm}$. Typical exposure doses of $65 \mathrm{~mJ} \mathrm{~cm}{ }^{-2}$ are used. This value is optimized for each pattern individually, depending on the fill factor and therefore the transmittance of the mask. A post exposure bake $\left(110^{\circ} \mathrm{C}, 1 \mathrm{~min}\right)$ is performed after the exposure, followed by puddle development in $\mathrm{AZ}^{\circledR}$ MIF 327 developer (1 min).

Our mask-aligner in use has an Etel SARIGAN* movement stage, for high-precision positioning of the photoresist-coated wafer relative to the photomask. The proximity gap of $20 \mu \mathrm{m}$, i.e. the distance between mask and wafer, is controlled using an AvANTES ${ }^{\dagger}$ spectroscopic reflectrometry setup at three locations.

Figure 4 shows SEM micrographs of square and hexagonal arrays of circles as well as a square array of squares. The period of the structures is $1.39 \mu \mathrm{m}$ in Figs. $4 \mathrm{a}$ and $4 \mathrm{~b}$, and $1.97 \mu \mathrm{m}$ in Fig. 4c. The latter period equals half the

*ETEL S.A., CH-2112 Môutier, Switzerland, etel.ch, etel@etel.ch

${ }^{\dagger}$ Avantes BV, NL-7333 NS Apeldoorn, The Netherlands, avantes.com, info@avantes.com
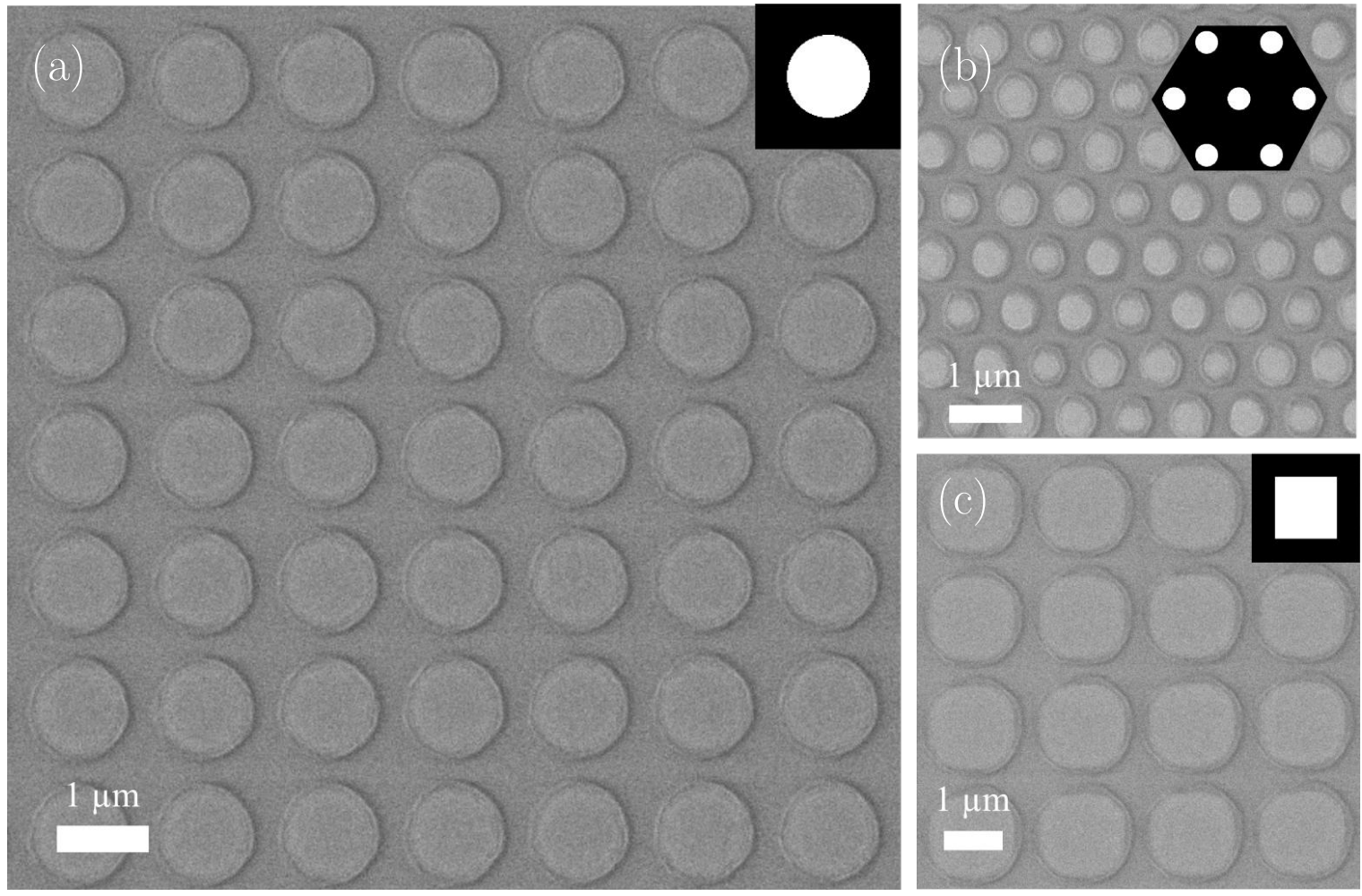

Figure 4: Scanning electron microscope (SEM) micrographs of (a) circles, (b) a hexagonal array of circles (period $1.39 \mu \mathrm{m}$ ), and (c) squares (period $1.97 \mu \mathrm{m}$ ). The insets show the mask layout. The periodic structure and the shape of the patterns are replicated in the resist, while the size of the features can be adapted by the exposure dose. 
Talbot distance $\left(n=\frac{1}{2}\right)$, where the pattern is self-imaged, shifted by half a period. The replication of the mask features is clearly visible, with uniform size distribution over the entire periodic array (size $800 \mu \mathrm{m} \times 800 \mu \mathrm{m}$ ). The size of the individual elements can be controlled via the exposure dose. A subsequent transfer of the patterns to the substrate is possible using a hard etch mask, as demonstrated for example in Ref. 5.

\section{COMPARISON OF I-LINE AND DUV TALBOT LITHOGRAPHY}

When comparing Talbot lithography with i-line illumination at $365 \mathrm{~nm}$ and the DUV laser source at $193 \mathrm{~nm}$ presented here, several aspects can be highlighted. First, to obtain the same pattern period with i-line illumination, the proximity gap has to be approximately halved, to $10.4 \mu \mathrm{m}$ instead of $20 \mu \mathrm{m}$. At the same time, the depth of focus (DOF) is about halved ${ }^{21}$ rendering an exact setting of the proximity gap more critical.

The high absorption of the resist in the DUV, compared to typical i-line resists, limits the resist thickness to well below $1 \mu \mathrm{m}$ (120 nm in our case). As a consequence, the high DOF can not be exploited to achieve high aspect ratios in the resist, but rather to reduce the sensitivity to variations in the proximity gap.

The high brilliance of the light source allows efficient beam shaping, i.e. a high conversion efficiency of the light into plane wave illumination. In the current optical setup, approximately one tenth of the laser output power ends up in the plane wave illumination. Further improvement can be reached by using optical elements with anti-reflective coatings. Mask-aligners a with conventional i-line illumination source as high-pressure mercury arc lamps typically use MO Exposure Optics ${ }^{\circledR 26,27}$ to reach plane wave illumination. While excellent spatial and angular uniformity of the illumination has been demonstrated, ${ }^{27}$ this requires to block a large share of the power by an illumination filter plate (IFP), rendering the beam shaping inefficient. Recently, a novel approach for efficiently shaping the angular spectrum of laser illumination in mask-aligner lithography has been presented, ${ }^{2}$ relying on a galvanometer scanner to define the angular spectrum.

\section{CONCLUSIONS AND OUTLOOK}

Using the novel concept of a CW laser emitting at a wavelength of $193 \mathrm{~nm}$, we were able to demonstrate its applicability to Talbot mask-aligner lithography. The continuous operation, the small volume of the system, and the avoidance of hazardous gases are the advantages of our laser source over conventional DUV Excimer laser systems. All these aspects lead to a straightforward implementation into a mask-aligner setup.

Efficient beam shaping has been demonstrated, relying on an imaging homogenizer setup. The main optical element is a Köhler integrator, consisting of a microlens array (MLA) and a Fourier lens. A single rotating diffuser is sufficient to avoid the emergence of interference effects, which arise from the high spatial and temporal coherence of the illuminating laser source. Uniform plane wave illumination over square fields in the mask plane with sizes of up to $15 \mathrm{~mm} \times 15 \mathrm{~mm}$ and an angular spectrum of less than $1^{\circ}$ are shown.

Periodic structures with a period down to $1.4 \mu \mathrm{m}$ were printed at a proximity gap of $20 \mu \mathrm{m}$ using a chromium amplitude mask. Such a large proximity gap relaxes the requirements imposed on the mask-aligner setup in terms of mechanical stability and accuracy, the demands on the wafer flatness, and increase the process window due to an enlarged depth of focus (DOF).

While keeping the proximity gap constant, the period of the lithographic pattern can be further reduced by using a higher Talbot plane $(n>1)$. In this scenario, the dimensions of the structure have to be chosen such that a higher Talbot plane coincides with the wafer plane. Crucially, the plane wave illumination has to possess a sufficient contrast in order to allow a high-quality reproduction of the mask features.

Further improvement in the laser output power promises to extend the field sizes up to full wafer scale, while keeping the exposure times comparable to i-line illumination. The combination of a frequency-quadrupled $\mathrm{CW}$ diode laser source emitting at a wavelength of $193 \mathrm{~nm}$ with the Talbot effect proofs to be a viable way for pattern transfer in mask-aligner proximity lithography and a serious alternative to Excimer light sources. 


\section{ACKNOWLEDGMENTS}

The authors want to thank J. Béguelin and S. Tournois for useful discussions and technical support. Furthermore, the authors acknowledge the preparation of KBBF crystals by R. Li, X. Wang, and L. Liu. A.V. acknowledges support by the Karlsruhe School of Optics and Photonics (KSOP).

We received funding from the KMU-innovativ program, project UVMOPA (FKZ13N13022). This project has received funding from the European Union's Horizon 2020 research and innovation programme under the Marie Skłodowska-Curie grant agreement No 675745.

\section{REFERENCES}

[1] Partel, S., Zoppel, S., Hudek, P., Bich, A., Vogler, U., Hornung, M., and Voelkel, R., "Contact and proximity lithography using 193nm Excimer laser in Mask Aligner," Microelectronic Engineering 87(5-8), 936 (2010).

[2] Weichelt, T., Bourgin, Y., and Zeitner, U. D., "Mask aligner lithography using laser illumination for versatile pattern generation," Opt. Express 25(18), 20983 (2017).

[3] Scholz, M., Opalevs, D., Leisching, P., Kaenders, W., Wang, G., Wang, X., Li, R., and Chen, C., "A bright continuous-wave laser source at 193 nm," Applied Physics Letters 103(5), 051114 (2013).

[4] Opalevs, D., Scholz, M., Gilfert, C., Li, R., Wang, X., Liu, L., Vetter, A., Kirner, R., Scharf, T., Noell, W., Rockstuhl, C., Voelkel, R., and Leisching, P., "Semiconductor-based narrow-line and high-brilliance 193nm laser system for industrial applications," presented at SPIE Photonics West 2018, Paper 10511-83 (2018).

[5] Kirner, R., Vetter, A., Opalevs, D., Gilfert, C., Scholz, M., Leisching, P., Scharf, T., Noell, W., Rockstuhl, C., and Voelkel, R., "Mask-aligner lithography using a continuous-wave diode laser frequency-quadrupled to $193 \mathrm{~nm}, "$ Optics Express 26(2), 730 (2018).

[6] Talbot, H., "LXXVI. Facts relating to optical science. No. IV," Philosophical Magazine Series 3 9(56), 401-407 (1836).

[7] Rayleigh, L., "XXV. On copying diffraction-gratings, and on some phenomena connected therewith," Philosophical Magazine Series 5 11(67), 196-205 (1881).

[8] Stuerzebecher, L., Fuchs, F., Zeitner, U. D., and Tuennermann, A., "High-resolution proximity lithography for nano-optical components," Microelectronic Engineering 132, 120-134 (2015).

[9] Rothschild, M., "A review of excimer laser projection lithography," Journal of Vacuum Science \& Technology B: Microelectronics and Nanometer Structures 6(1), 1 (1988).

[10] Patorski, K., "The Self-Imaging Phenomenon and its Applications," Progress in Optics 27, 1-108, Elsevier (1989).

[11] Zanke, C., Qi, M., and Smith, H. I., "Large-area patterning for photonic crystals via coherent diffraction lithography," Journal of Vacuum Science \& Technology B: Microelectronics and Nanometer Structures 22(6), 3352 (2004).

[12] Isoyan, A., Jiang, F., Cheng, Y. C., Cerrina, F., Wachulak, P., Urbanski, L., Rocca, J., Menoni, C., and Marconi, M., "Talbot lithography: Self-imaging of complex structures," Journal of Vacuum Science 83 Technology B: Microelectronics and Nanometer Structures 27(6), 2931 (2009).

[13] Kim, M.-S., Scharf, T., Menzel, C., Rockstuhl, C., and Herzig, H. P., "Talbot images of wavelength-scale amplitude gratings," Optics Express 20(5), 4903 (2012).

[14] Dunbar, L. A., Nguyen, D., Timotijevic, B., Vogler, U., Veseli, S., Bergonzi, G., Angeloni, S., Bramati, A., Voelkel, R., and Stanley, R. P., "Talbot lithography as an alternative for contact lithography for submicron features," Proc. SPIE 8974 (2014).

[15] Solak, H. H., Dais, C., and Clube, F., "Displacement Talbot lithography: a new method for high-resolution patterning of large areas," Optics Express 19(11), 10686 (2011).

[16] Chang, C.-H., Tian, L., Hesse, W. R., Gao, H., Choi, H. J., Kim, J.-G., Siddiqui, M., and Barbastathis, G., "From Two-Dimensional Colloidal Self-Assembly to Three-Dimensional Nanolithography," Nano Letters 11(6), 2533-2537 (2011).

[17] Karim, W., Tschupp, S. A., Oezaslan, M., Schmidt, T. J., Gobrecht, J., van Bokhoven, J. A., and Ekinci, Y., "High-resolution and large-area nanoparticle arrays using EUV interference lithography," Nanoscale 7(16), 7386-7393 (2015). 
[18] Berry, M. V. and Klein, S., "Integer, fractional and fractal Talbot effects," Journal of Modern Optics 43(10), 2139-2164 (1996).

[19] Luo, K.-H., Wen, J., Chen, X.-H., Liu, Q., Xiao, M., and Wu, L.-A., "Second-order Talbot effect with entangled photon pairs," Physical Review A 80(4), 043820 (2009).

[20] Song, X.-B., Wang, H.-B., Xiong, J., Wang, K., Zhang, X., Luo, K.-H., and Wu, L.-A., "Experimental Observation of Quantum Talbot Effects," Physical Review Letters 107(3), 033902 (2011).

[21] Born, M., Wolf, E., Bhatia, A. B., Clemmow, P. C., Gabor, D., Stokes, A. R., Taylor, A. M., Wayman, P. A., and Wilcock, W. L., [Principles of Optics: Electromagnetic Theory of Propagation, Interference and Diffraction of Light], Cambridge University Press, 7 ed. (1999).

[22] Voelkel, R. and Weible, K. J., "Laser beam homogenizing: limitations and constraints," Proc. SPIE 7102 (2008).

[23] Drever, R. W. P., Hall, J. L., Kowalski, F. V., Hough, J., Ford, G. M., Munley, A. J., and Ward, H., "Laser phase and frequency stabilization using an optical resonator," Applied Physics B Photophysics and Laser Chemistry 31(2), 97 (1983).

[24] Goodman, J. W., [Speckle Phenomena in Optics : Theory and Applications], Roberts \& Co (2007).

[25] Kirner, R., Vetter, A., Opalevs, D., Scholz, M., Leisching, P., Scharf, T., Noell, W., Rockstuhl, C., and Voelkel, R., "Enabling proximity mask-aligner lithography with a $193 \mathrm{~nm} \mathrm{CW}$ light source," presented at SPIE Advanced Lithography 2018, Paper 10587-50 (2018).

[26] Voelkel, R., Vogler, U., Bich, A., Weible, K. J., Eisner, M., Hornung, M., Kaiser, P., Zoberbier, R., and Cullmann, E., "Illumination system for a microlithographic contact and proximity exposure apparatus," European Patent 2253997 A2 (2009).

[27] Voelkel, R., Vogler, U., Bich, A., Pernet, P., Weible, K. J., Hornung, M., Zoberbier, R., Cullmann, E., Stuerzebecher, L., Harzendorf, T., and Zeitner, U. D., "Advanced mask aligner lithography: new illumination system," Optics Express 18(20), 20968 (2010). 